\title{
Construction Prototyping, Flight Dynamics Modeling, and Aerodynamic Analysis of Hybrid VTOL Unmanned Aircraft
}

\author{
Roman Czyba (D), ${ }^{1}$ Marcin Lemanowicz, ${ }^{2}$ Zbigniew Gorol, ${ }^{3}$ and Tomasz Kudala ${ }^{3}$ \\ ${ }^{1}$ Faculty of Automatic Control, Electronics and Computer Science, Department of Automatic Control, Silesian University of Technology, \\ 16 Akademicka St., Gliwice 44-100, Poland \\ ${ }^{2}$ Faculty of Chemistry, Department of Chemical Engineering and Process Design, Silesian University of Technology, 16 Akademicka St., \\ Gliwice 44-100, Poland \\ ${ }^{3}$ Faculty of Mechanical Engineering, Silesian University of Technology, 16 Akademicka St., Gliwice 44-100, Poland
}

Correspondence should be addressed to Roman Czyba; roman.czyba@polsl.pl

Received 17 July 2018; Accepted 30 August 2018; Published 1 October 2018

Academic Editor: Jose E. Naranjo

Copyright (C) 2018 Roman Czyba et al. This is an open access article distributed under the Creative Commons Attribution License, which permits unrestricted use, distribution, and reproduction in any medium, provided the original work is properly cited.

\begin{abstract}
A challenging issue associated with fixed-wing Unmanned Aerial Vehicles (UAVs) is that these vehicles are often not appropriate for operating effectively in limited airspace. This problem emerges especially in urban environment where the usage of a runway is not possible, and UAVs usually have to fly at a relatively low speed and altitude. The development of a vertical take-off and landing (VTOL) fixed-wing plane is a promising trend which hopefully will solve this issue. This paper presents the design process of an unmanned vertical take-off and landing aircraft including prototyping of the airframe construction and mathematical modeling as well as computational fluid dynamics (CFD) simulations. The designed system is to be a hybrid platform, for which different operating modes correspond to the vertical flight, transition, and spatial flight in the airframe system. The paper discusses an iterative design process of the platform with emphasis on CAD design and aerodynamic analysis for particular flight modes. The operating prototype is presented and future plans for platform improvement are discussed.
\end{abstract}

\section{Introduction}

Aircraft technology has been developing for over a century since the Wright brothers built their first manned plane, through Sperry's autopilot, until the 21st century when the Unmanned Aerial Vehicles (UAVs) were invented [17]. Generally, there are three design structures: fixed-wing aircraft, rotorcrafts, and aerostats. However, there are many innovative solutions in the form of aforementioned hybrid constructions, giving interesting flight properties. Each type of UAV has its own advantages that predispose it to perform specific tasks. Many UAVs [8] such as MQ9-Reaper, RQ-2 Pioneer, RQ-5 Hunter, and RQ-4 Global Hawk were designed as fixed-wing type, and their advantage is flight duration and thereby the area of operational activities. Their disadvantage is the need to take-off and land from the runway, which significantly limits their functionality. Another critical problem associated with fixed-wing UAVs is that these vehicles are often not appropriate for operating effectively in limited airspace. This is evident in urban environment where the usage of a runway is not possible, and UAVs usually have to fly at a relatively low speed and altitude. This "U-Space" [9] covers altitude up to 150 meters in the European Union and is an area for the development of a strong and dynamic drone services market [10]. In turn, multirotors [11, 12] have the capability of vertical take-off and landing (VTOL), which increases their operational flexibility. But this construction is unfortunately associated with a high energy demand, which makes the flight relatively short. A promising trend is development of a vertical take-off and landing (VTOL) fixedwing plane or so-called hybrid UAV or VTOL aircraft, which combines the advantages of both designs.

Over the last five years, many hybrid VTOL structures have been created $[1,13-16]$. One of them is Tilt-Rotor [1720], where multiple rotors are mounted on rotating shafts or nacelles. Another solution is the Tilt-Wing concept, where 


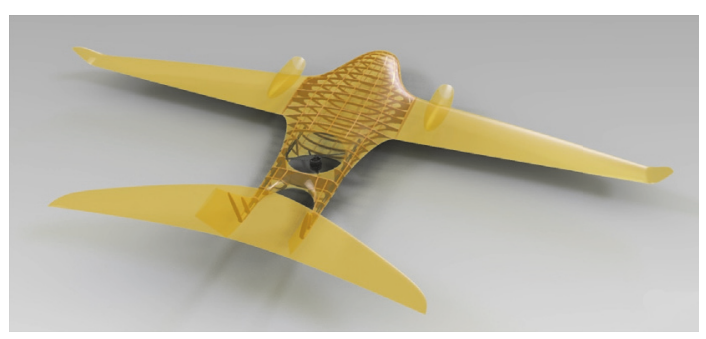

FIgURE 1: The initial concept of the VTOL UAV.

the transition between individual flight phases is obtained by tilting the wings with fixed rotors [17, 21, 22]. Unfortunately, during take-off, landing, and hovering, the wings will be directed upwards which makes the aircraft more susceptible to transverse winds. Another type of convertiplane is Dual-System [23], where multiple rotors are always directed upwards for vertical flight and another separate rotor for horizontal flight. This configuration is very simple to apply in terms of design and modeling because two flight phases could be analysed separately. But for most of the mission, the platform must transport unnecessary mass, which can be replaced by fuel or batteries. Another solution is a configuration of Tail-Sitter $[24,25]$. This platform takes off and lands vertically on its tail and the whole aircraft tilts forward using differential thrust or control surfaces to achieve horizontal flight. In conclusion, it is true that today VTOL aircraft exist and operate in practice, but they are burdened with various limitations and disadvantages.

In this paper, a novel design configuration in a form of hybrid of conventional aircraft and quadrotor, with two of four rotors mounted in nacelles with ability of rotation, has been proposed. It will have a unique feature among similar solutions-a thrust vectoring of engines that ensures their usage in both vertical and horizontal flight. Compared to the above-mentioned designs, an advantage of proposed approach is an ability to rotate only the rotors themselves, not the whole wing. During take-off, landing, and hovering, this solution makes the aircraft more robust to cross winds. In the airplane mode, the advantage is also the usage of rotors in the pushing system behind the trailing edge of the wing, which ensures uniformity of the air stream flowing around the airfoil. In turn, in VTOL mode, the usage of pushing propeller from underside of the rotor increases the efficiency of the propulsion system $[26,27]$.

The main purpose of this paper is to present the concept of VTOL aircraft, from scratch to final implementation, covering the individual stages of prototyping and then deriving a mathematical model based on the physical model and computational fluid dynamics CFD simulations [28]. A mathematical model that can accurately describe the flight dynamics is exceedingly important in the design of a flight control system. In the case of hybrid UAVs, the whole flight can be divided into three phases, namely, vertical flight mode, transition mode, and horizontal flight mode. As a consequence, the development of a real flight dynamics model is much more difficult compared to the conventional UAVs and causes many problems.
The paper is organized as follows. First, the introduction is provided in Section 1. Then, the concept of VTOL aircraft and construction prototyping are presented in Section 2. The mechanical design and an explanation of flight phases are shown in Section 3. A mathematical model including forces and moments definition in individual flight phases are determined in Section 4. Next, the computational fluid dynamics (CFD) simulations are described together with a discussion of received results. The conclusions are briefly discussed in the last section.

\section{Mechanical Design and Construction Prototyping}

The initial concept of the UAV assumed that the construction should be efficient in terms of aerodynamics as far as it is possible. Therefore the parts of the platform which are necessary for the hovering flight mode (especially engines and propellers) should not generate drag during standard flight mode which means that they should be hidden within fuselage. Simultaneously, the minimum number of support points which provides stable UAV hovering without necessity of applying any advanced and complicated control mechanisms is equal to three. In the light of these assumptions the obvious choice was a platform configuration with two engines mounted in the front of the wings and a ducted propeller placed within the fuselage (Figure 1).

The front engines together with propellers were designed to tilt from vertical orientation used in standard flight mode to horizontal orientation used in hovering mode. Together with the ducted propeller they were arranged on a circular plan whose centre was located at the mass centre of construction. The advantage of such construction solution was the possibility of hiding the fuselage propeller during standard flight mode and as a result to minimize generated drag. On the other hand the placement of the fuselage propeller complicated significantly the tail construction. Moreover, the size of the fuselage had to be increased in order to accommodate the mechanical construction. As a result the aerodynamic properties of the platform were not satisfactory. It was characterized by low lift with simultaneous high drag. Moreover, the thrust generated by fuselage propeller was too low and its mechanical construction was unnecessarily complicated. In the initial design two separate autopilots were considered both for hovering and standard flight modes connected via mixer. To conclude, the concept with three 


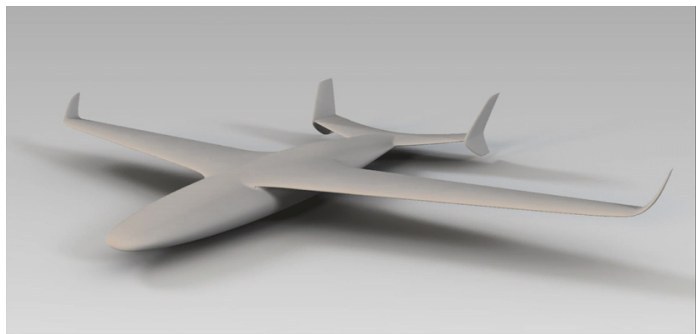

FIGURE 2: The second concept of the VTOL UAV.

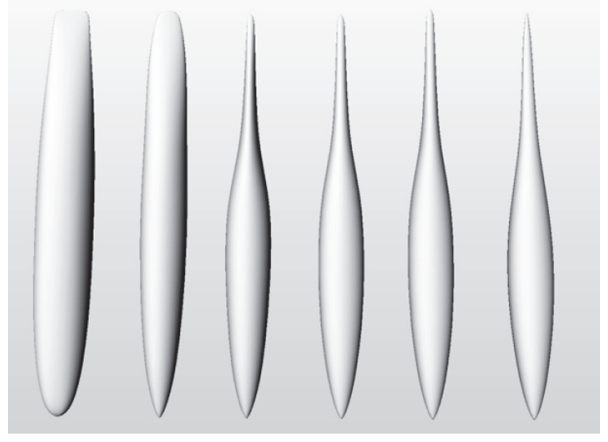

FIGURE 3: The optimization process of fuselage.

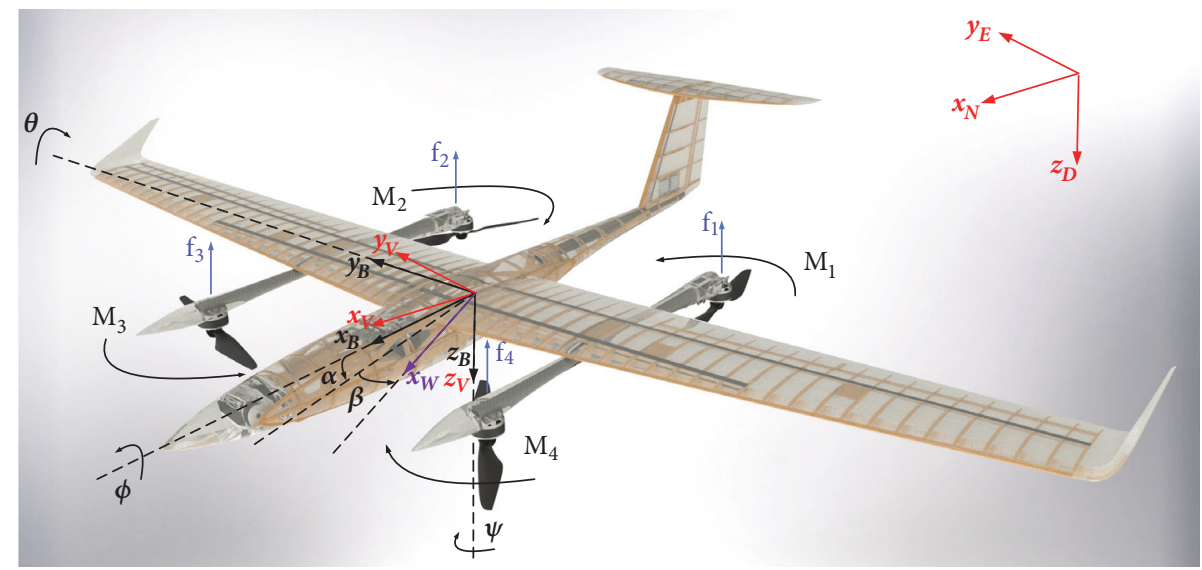

FIgURE 4: The final design of the VTOL aircraft.

supporting points was rejected. The disadvantages resulting from the placement of the third propeller overshadowed any advantages of this solution.

The second iteration of the concept design was based on four points of support (Figure 2). The first two engines with propellers were placed in front of the wings as in the previous design. Once again their tilt depended on the flight mode. However, another two propellers were drawn out from the fuselage on specially designed arms. Due to the elimination of the ducted propeller the size of fuselage decreased. Moreover, the design of the tail changed from double "T" configuration into " $\mathrm{H}$ " configuration.

During the design process two different plane configurations were considered, i.e., centre-wing and high-wing. Moreover, the shape of the fuselage was optimized (Figure 3).
The biggest advantage of this design was the ease to control hovering mode of flight. The control characteristics were identical to the characteristics of common quadcopter platforms. On the other hand, still the aerodynamic properties of the UAV were not satisfactory. There were also some technical issues with moving arms for hovering mode. After construction and tests of a prototype the third iteration of design started.

In the third and final iteration of design the wing profile was changed. Moreover, the construction of the tail was simplified further from " $\mathrm{H}$ " configuration into single " $\mathrm{T}$ " configuration with floating horizontal stabilizer (Figure 4). All four rotors were placed on two beams mounted below wings which resulted in " $\mathrm{H}$ " propulsion configuration. In the hovering flight mode all rotors were directed downward. 


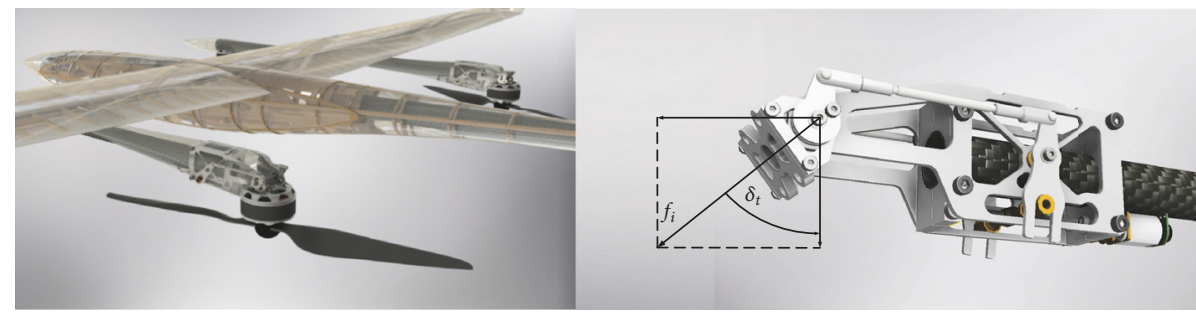

FIgURE 5: Tilt mechanism.
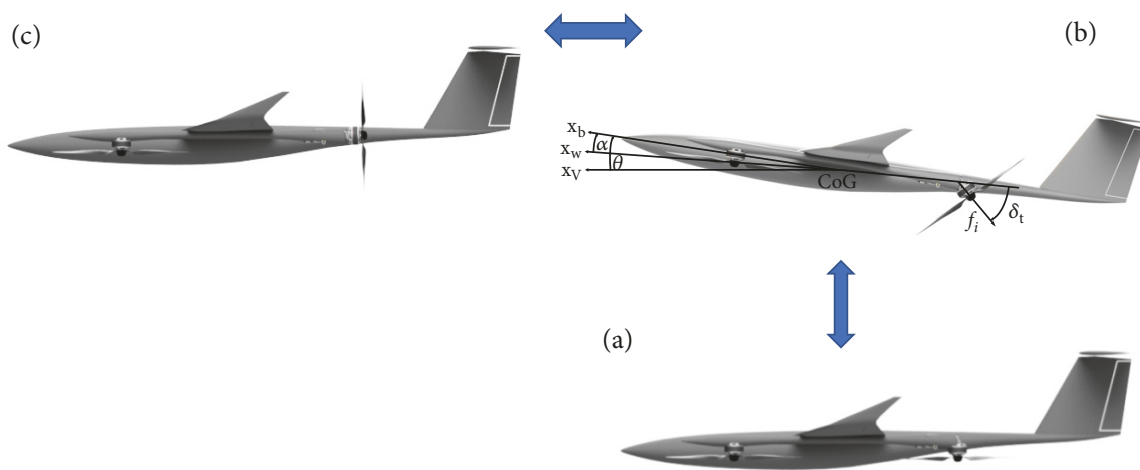

FIgURE 6: The flight envelope: (a) VTOL mode, (b) transition mode, and (c) aircraft mode.

In the transition mode the rear propellers $\left(\mathrm{M}_{1}, \mathrm{M}_{2}\right)$ tilted into vertical position whereas the front rotors $\left(\mathrm{M}_{3}, \mathrm{M}_{4}\right)$ were gradually turned off. In the standard flight mode the special construction of front propellers allowed them to fold due to action of drag. This resulted in the overall drag reduction. The downward orientation of propellers as well as placement of vertical propellers behind the wing resulted from aerodynamic optimization. Comparing upward configuration, downward configuration is characterized with higher efficiency $[26,27]$ and the pushing configuration of propulsion system does not influence the airflow over wing profile.

\section{VTOL Aircraft Design}

The developed VTOL prototype (Figure 4) allows vertical take-off, transition to forward flight, travel to the destination in cruise flight, and transition back to hover flight as well as landing vertically. Finally, the proposed design configuration is a hybrid of conventional aircraft in the high-wing system and quadrotor, with two of four rotors mounted in nacelles and having the ability to rotate.

The transition mechanisms (Figure 5), which are applied to achieve the conversion from vertical flight to horizontal flight and vice versa, are based on the fact that rear rotors $\left(M_{1}\right.$, $\mathrm{M}_{2}$ ) are mounted on rotating nacelles. During transition, the rotors tilt gradually towards flight direction providing the aircraft forward speed. Their interaction with the front rotors $\left(\mathrm{M}_{3}, \mathrm{M}_{4}\right)$ ensures flight at a fixed height.

The flight envelope of the UAV platform is divided into the three following flight modes, which was achieved by means of the collective angular displacement of the two rear rotors (see Figure 6):

(i) VTOL mode: this flight phase is intended for vertical climbing, descending, and hovering. The flying platform works in a multirotor mode and the whole thrust is derived from the four rotors. To minimize power consumption, after reaching the required minimum altitude, the UAV platform immediately proceeds to flight in the aircraft mode.

(ii) Transition mode: two rear propellers are tilted synchronously towards the vertical direction, which in turn causes the horizontal speed of the aircraft to increase. The angular position is controlled by changing the thrust of particular rotors so that the airframe obtains a given angle of attack relative to the incoming air streams. With the increase of the horizontal speed, the fixed wings develop lift. Before the angle of attack reaches a given value, the roll angle is controlled by the difference in thrust between the left and right rotors. Yaw angle is regulated by the counteractive moments generated by pairs of rotors: M1, M3 and M2, M4. In turn, pitch angle is controlled by the difference in thrust between the front and rear rotors. For horizontal-to-hover transition the reverse procedure is performed.

(iii) Aircraft mode: at this flight phase the UAV has a sufficient speed of translational movement, which allows generating lift force. Under such conditions the platform behaves like a conventional airplane, and aerodynamic control surfaces, such as rudder, 


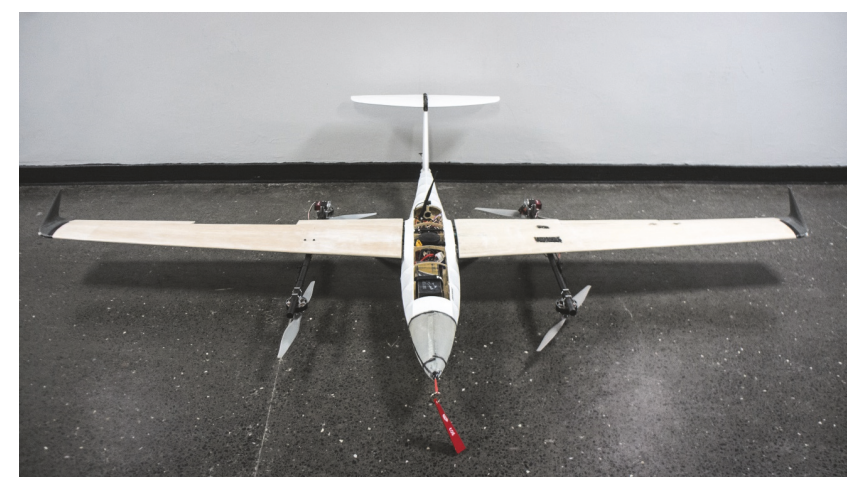

FIGURE 7: VTOL aircraft.

elevator, and ailerons, provide yaw, pitch, and roll motions. The required thrust force is generated by two rear rotors $\left(M_{1}, M_{2}\right)$, tilted by an angle of 90 degrees in relation to the initial position. The other two front motors $\left(\mathrm{M}_{3}, \mathrm{M}_{4}\right)$ are turned off and remain in initial position. This flight phase is intended for energy efficient long term operations.

Such structure is characterized by high maneuverability in a limited area, and the flight time is relatively long above one hour, which is unachievable in the case of conventional multirotor carriers. The advantage of proposed approach is an ability to rotate only the rotors themselves, not the whole wing. During take-off, landing, and hovering, this solution makes that the aircraft more robust to cross winds. In the airplane mode, the advantage is also the usage of rotors in the pushing system behind the trailing edge of the wing flap, which ensures uniformity of the air stream flowing around the airfoil. The front engines are switched off, because they only disturb the flow of air streams, without significantly increasing the lift force generated on the wing. In turn, in VTOL mode, the usage of pushing propeller from underside of the rotor increases the efficiency of the propulsion system $[26,27]$.

The technical specification of the designed and constructed aircraft is included in Table 1.

To ensure smooth switching between flight modes, a certain error margin was provided, as shown in Table 2.

Figure 7 shows the final realization of the VTOL aircraft.

\section{Mathematical Modeling}

The configuration of VTOL airplane is shown in Figure 4. In this paper $x, y$, and $z$ axes of the inertial coordinate system are defined as a right handed Cartesian coordinate system with North, East, and Downward directions, abbreviated as the NED coordinate system. The movement of the aircraft will be considered in relation to the following coordinates systems [29]:

(i) inertial reference frame $F_{E}\left(x_{N}, y_{E}, z_{D}\right)$ with the origin determined on the surface of the Earth,

(ii) inertial reference frame $F_{V}\left(x_{V}, y_{V}, z_{V}\right)$ with the origin in the centre of the mass of the aircraft,
TABLE 1: VTOL aircraft specification.

\begin{tabular}{lc}
\hline Parameter & Value \\
\hline Configuration & VTOL aircraft \\
Wing span & $2520[\mathrm{~mm}]$ \\
Length & $1600[\mathrm{~mm}]$ \\
Wing area & $54\left[\mathrm{dm}^{2}\right]$ \\
Propeller diameter and pitch & $16 \times 8[\mathrm{inch}]$ \\
Minimum horizontal speed & $10[\mathrm{~m} / \mathrm{s}]$ \\
Maximum speed & $30[\mathrm{~m} / \mathrm{s}]$ \\
\hline
\end{tabular}

TABLE 2: VTOL flight modes.

\begin{tabular}{lcc}
\hline Flight Mode & Horizontal Speed & Vertical Speed \\
\hline VTOL & $0 \div 1 \mathrm{~m} / \mathrm{s}$ & $0 \div 5 \mathrm{~m} / \mathrm{s}$ \\
Transition & $0 \mathrm{~m} / \mathrm{s} \div 12 \mathrm{~m} / \mathrm{s}$ & $0 \mathrm{~m} / \mathrm{s} \div 1 \mathrm{~m} / \mathrm{s}$ \\
Aircraft & $10 \mathrm{~m} / \mathrm{s} \div 30 \mathrm{~m} / \mathrm{s}$ & $0 \mathrm{~m} / \mathrm{s} \div 5 \mathrm{~m} / \mathrm{s}$ \\
\hline
\end{tabular}

(iii) body reference frame $F_{B}\left(x_{B}, y_{B}, z_{B}\right)$,

(iv) wind reference frame $F_{w}\left(x_{w}, y_{w}, z_{w}\right)$.

Assuming that VTOL airplane is a rigid body with six degrees of freedom, the state of aircraft motion is defined by twelve coordinates. The following state vector is adopted:

$$
\bar{X}=[U, V, W, \phi, \theta, \psi, P, Q, R, x, y, z]^{T}
$$

where $U, V, W$ and $P, Q, R$ are projections of velocity and angular velocity onto the $x_{B}, y_{B}$, and $z_{B}$ axes of the body axis system $F_{B}$, respectively. Three Euler angles: roll angle $\phi$, pitch angle $\theta$, and yaw angle $\psi$ are introduced to determine the angular position of aircraft relatively to the Earth, i.e., the orientation of the body coordinate system $F_{B}$ with respect to the Earth coordinate system $F_{V}$.

Whereas the control vector contains the following variables, their appropriate combinations allow effective platform control in particular modes of flight:

$$
\bar{U}=\left[u_{1}, u_{2}, u_{3}, u_{4}, \delta_{t}, \delta_{e}, \delta_{r}, \delta_{a}\right]^{T}
$$

where $u_{i}$ are PWM motor inputs $(i=1, \ldots, 4), \delta_{t}$ is deflection of the rotor nacelle, $\delta_{e}$ is elevator deflection, $\delta_{r}$ is rudder deflection, and $\delta_{a}$ is ailerons deflection. 
Denoting the velocities as follows:

$\left(\bar{V}_{E}\right)_{F_{B}}=[U, V, W]^{T}:$ the aircraft velocity with respect to Earth,

$\left(\bar{V}_{\alpha}\right)_{F_{B}}=\left[V_{\alpha x}, V_{\alpha y}, V_{\alpha z}\right]^{T}:$ the aircraft velocity with respect to air,

$\left(\bar{V}_{W}\right)_{F_{W}}=\left[U_{W}, V_{W}, W_{W}\right]^{T}:$ the wind velocity with respect to Earth,

the relationship between those velocities can be written as

$$
\left(\bar{V}_{\alpha}\right)_{F_{B}}=\left(\bar{V}_{E}\right)_{F_{B}}-\boldsymbol{D}_{B W}\left(\bar{V}_{W}\right)_{F_{W}}
$$

In order to determine the aerodynamic forces and moments acting on the aircraft, it is necessary to know the flight speed and two angles: the angle of attack $\alpha$ and the sideslip angle $\beta$. These quantities are shown in Figure 4, which shows the flight of the airplane taking into account the flow of air streams from its left side. They are defined based on the coordinates velocity vector:

$$
\begin{aligned}
\alpha & =\arctan \frac{V_{\alpha z}}{V_{\alpha x}} \\
\beta & =\arcsin \frac{V_{\alpha y}}{V_{\alpha}} \\
\left(V_{\alpha}\right)_{F_{B}} & =\left(V_{\alpha x}^{2}+V_{\alpha y}^{2}+V_{\alpha z}^{2}\right)^{1 / 2}
\end{aligned}
$$

Before introducing the appropriate equations describing the VTOL aircraft movement, the transformation matrices which interconnect particular coordinate systems will be presented in the following form:

(i) The transformation matrix $\boldsymbol{L}_{B V}$ from the Earth coordinate system $F_{V}$ to the body coordinate system $F_{B}$ :

$$
L_{B V}=\left[\begin{array}{ccc}
\cos \theta \cos \psi & \cos \theta \sin \psi & -\sin \theta \\
\sin \phi \sin \theta \cos \psi-\cos \phi \sin \psi & \sin \phi \sin \theta \sin \psi+\cos \phi \cos \psi & \sin \phi \cos \theta \\
\cos \phi \sin \theta \cos \psi+\sin \phi \sin \psi & \cos \phi \sin \theta \sin \psi-\sin \phi \cos \psi & \cos \phi \cos \theta
\end{array}\right]
$$

(ii) The transformation matrix $\boldsymbol{D}_{B W}$ from the wind coordinate system $F_{W}$ to the body coordinate system $F_{B}$ :

$$
D_{B W}=\left[\begin{array}{ccc}
\cos \alpha \cos \beta & -\cos \alpha \sin \beta & -\sin \alpha \\
\sin \beta & \cos \beta & 0 \\
\sin \alpha \cos \beta & -\sin \alpha \sin \beta & \cos \alpha
\end{array}\right]
$$

(iii) The matrix $\boldsymbol{T}_{\omega}$ describes a relationship between the Euler angles and the angular velocity $P, Q, R$ of the aircraft:

$$
T_{\omega}=\left[\begin{array}{ccc}
1 & \sin \phi \frac{\sin \theta}{\cos \theta} & \cos \phi \frac{\sin \theta}{\cos \theta} \\
0 & \cos \phi & -\sin \phi \\
0 & \frac{\sin \phi}{\cos \theta} & \frac{\cos \phi}{\cos \theta}
\end{array}\right]
$$

In this paper, air movements with respect to the Earth are assumed to be zero, and the relationship between velocities takes the form:

$$
\left(\bar{V}_{\alpha}\right)_{F_{B}}=\left(\bar{V}_{E}\right)_{F_{B}}=\left(\begin{array}{c}
U \\
V \\
W
\end{array}\right)
$$

On this basis, the VTOL airplane movement is described by the following nonlinear system of twelve equations [29]:

$$
\begin{aligned}
m(\dot{U}+Q W-R V+g \sin \theta) & =F_{X} \\
m(\dot{V}+R U-P W-g \sin \phi \cos \theta) & =F_{Y}
\end{aligned}
$$

$$
\begin{aligned}
m(\dot{W}+P V-Q U-g \cos \phi \cos \theta) & =F_{Z} \\
\dot{P} I_{X}+Q R\left(I_{Z}-I_{Y}\right)-(P Q+\dot{R}) I_{X Z} & =L \\
\dot{Q} I_{Y}+P R\left(I_{X}-I_{Z}\right)+\left(P^{2}-R^{2}\right) I_{X Z} & =M \\
\dot{R} I_{Z}+P Q\left(I_{Y}-I_{X}\right)+(Q R-\dot{P}) I_{X Z} & =N \\
\left(\begin{array}{c}
\dot{\phi} \\
\dot{\theta} \\
\dot{\psi}
\end{array}\right) & =T_{\omega}\left(\begin{array}{c}
P \\
Q \\
R
\end{array}\right) \\
\left(\begin{array}{c}
\dot{x}_{E} \\
\dot{y}_{E} \\
\dot{z}_{E}
\end{array}\right) & =L_{B V}^{T}\left(\begin{array}{c}
U \\
V \\
W
\end{array}\right)
\end{aligned}
$$

where

$m$ is aircraft mass,

$g$ is gravitational acceleration,

$\bar{F}=\left[F_{X}, F_{Y}, F_{Z}\right]^{T}$ is force vector from aerodynamic forces and thrust,

$\bar{M}=[L, M, N]^{T}$ is torque vector from aerodynamic forces and thrust,

$I=\left[\begin{array}{ccc}I_{x} & 0 & -I_{x z} \\ 0 & I_{y} & 0 \\ -I_{x z} & 0 & I_{z}\end{array}\right]$ is inertia matrix.

Equations (11)-(18) allow describing spatial motion of aircraft in a body frame. 
4.1. Definition of Forces and Moments. The forces and moments at the aircraft centre of gravity have components due to aerodynamic effects and to engines' thrust (these components will be denoted, respectively, by the subscripts $A$ and $T$ ). The forces acting on the aircraft can be presented in the form of the following vector equation expressed in the $F_{B}$ system:

$$
\begin{aligned}
\bar{F}_{B} & =\left[\begin{array}{c}
F_{X} \\
F_{Y} \\
F_{Z}
\end{array}\right]=\bar{F}_{B_{T}}+\bar{F}_{B_{A}}=\left[\begin{array}{c}
F_{X_{T}} \\
F_{Y_{T}} \\
F_{Z_{T}}
\end{array}\right]+\left[\begin{array}{c}
F_{X_{A}} \\
F_{Y_{A}} \\
F_{Z_{A}}
\end{array}\right] \\
& =\left[\begin{array}{c}
F_{X_{T}} \\
0 \\
F_{Z_{T}}
\end{array}\right]+D_{B W}\left[\begin{array}{c}
-D \\
Y \\
-L
\end{array}\right]
\end{aligned}
$$

The thrust component $F_{Y T}$ can be produced by unbalanced engine power in a multiengine aircraft. However, in our case the applied thrust vectorization, by using servos in the rear gondolas, eliminates this problem $\left(F_{Y T}=0\right)$.

In general form, the thrust component $\bar{F}_{B_{T}}$ has the following form:

$$
\bar{F}_{B_{T}}=\left[\begin{array}{c}
F_{X_{T}} \\
0 \\
F_{Z_{T}}
\end{array}\right]=\left[\begin{array}{c}
f_{1} \cos \delta_{t}+f_{2} \cos \delta_{t} \\
0 \\
f_{3}+f_{4}+f_{1} \sin \delta_{t}+f_{2} \sin \delta_{t}
\end{array}\right]
$$

where $\delta_{t}$ is tilt-rotor deflection and $f_{i}$ is $i$ th rotor force $(i=$ $1, \ldots, 4)$.

In the simplest form the relationship between the thrust $f_{i}$ generated by the $i$ th rotor and control signal $u_{i}$ has been described as a square of the angular rotor velocity [30, 31]. A more detailed description, taking into account dynamics of BLDC motor, propeller aerodynamics, Electronic Speed Controller (ESC), and batteries, has been presented in [32]. For the purposes of this paper it was assumed that $f_{i}=f\left(u_{i}\right)$, where $i=1, \ldots, 4$.

The aerodynamic forces $\bar{F}_{B_{A}}$ acting on the aircraft are defined in terms of the dimensionless aerodynamic coefficients $[29,33]$ :

$$
D=\bar{q} S C_{D}-\text { drag }
$$

$$
\begin{aligned}
Y & =\bar{q} S C_{Y}-\text { sideforce } \\
L & =\bar{q} S C_{L}-\mathrm{lift}
\end{aligned}
$$

where $\bar{q}=0.5 \rho V_{\alpha}^{2}$ is free-stream dynamic pressure; $\rho$ is air density; $V_{\alpha}$ is aircraft velocity with respect to air; $S$ is wing area; $C_{D}, C_{Y}$, and $C_{L}$ are dimensionless force coefficients.

The moments acting on the aircraft during the flight in the $F_{B}$ system have the following form:

$$
\bar{M}=\left[\begin{array}{c}
L \\
M \\
N
\end{array}\right]=\bar{M}_{T}+\bar{M}_{A}=\left[\begin{array}{c}
l_{T} \\
m_{T} \\
n_{T}
\end{array}\right]+\left[\begin{array}{c}
l_{A} \\
m_{A} \\
n_{A}
\end{array}\right]
$$

Torque component resulting from the engine thrust $\bar{M}_{T}$ consists of the action of the thrust difference of each pair and from the gyroscopic effect:

$$
\begin{aligned}
\bar{M}_{T}= & {\left[\begin{array}{c}
l_{T} \\
m_{T} \\
n_{T}
\end{array}\right] } \\
= & {\left[\begin{array}{c}
F_{Z_{T}} \Delta y \\
F_{Z_{T}} \Delta x \\
F_{X_{T}} \sqrt{\Delta x^{2}+\Delta y^{2}}
\end{array}\right]+\omega } \\
& \times\left[\begin{array}{c}
-J_{r}\left(\Omega_{1}-\Omega_{2}\right) \cos \delta_{t} \\
0 \\
-J_{r}\left(\Omega_{1} \sin \delta_{t}+\Omega_{3}-\Omega_{2} \sin \delta_{t}-\Omega_{4}\right)
\end{array}\right]
\end{aligned}
$$

where

$\triangle x, \Delta y$ are distances from the force component to the axis of rotation,

$\omega=\left[\begin{array}{ccc}0 & -r & q \\ r & 0 & -p \\ -q & p & 0\end{array}\right]$

$J_{r}$ is rotor inertia,

$\Omega_{i}(i=1,2,3,4)$ is angular speed of $i$ th rotor.

Taking into account the geometric arrangement of each propulsion unit in the considered airframe (Figure 8), the following relationship was obtained:

$$
\bar{M}_{T}=\left[\begin{array}{c}
\frac{1}{2} l_{2} f_{4}+\frac{1}{2} l_{2} f_{1} \sin \delta_{t}-\frac{1}{2} l_{2} f_{3}-\frac{1}{2} l_{2} f_{2} \sin \delta_{t} \\
\frac{1}{2} l_{1} f_{3}+\frac{1}{2} l_{1} f_{4}-\frac{1}{2} l_{1} f_{2} \sin \delta_{t}-\frac{1}{2} l_{1} f_{1} \sin \delta_{t} \\
\sqrt{\left(\frac{1}{2} l_{1}\right)^{2}+\left(\frac{1}{2} l_{2}\right)^{2}} f_{1} \cos \delta_{t} \sin \delta_{g}-\sqrt{\left(\frac{1}{2} l_{1}\right)^{2}+\left(\frac{1}{2} l_{2}\right)^{2}} f_{2} \cos \delta_{t} \sin \delta_{g}
\end{array}\right]
$$




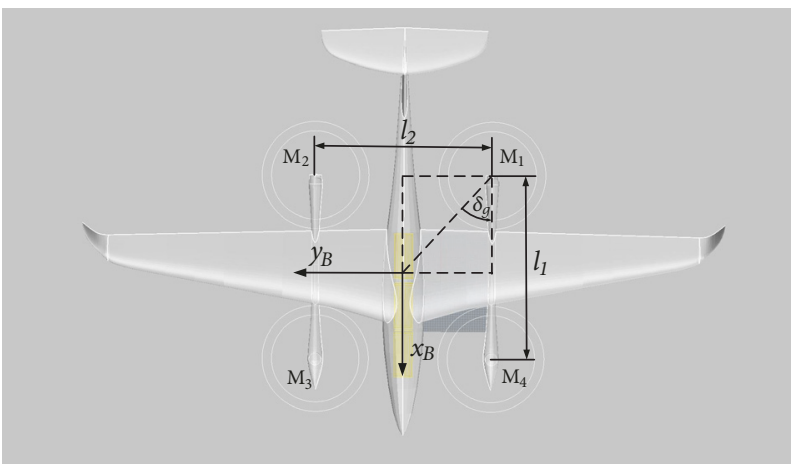

FIgURE 8: Configuration of the propulsion system.

$$
+\left[\begin{array}{c}
-J_{r} q\left(\Omega_{1} \sin \delta_{t}+\Omega_{3}-\Omega_{2} \sin \delta_{t}-\Omega_{4}\right) \\
-J_{r} r\left(\Omega_{1}-\Omega_{2}\right) \cos \delta_{t}+J_{r} p\left(\Omega_{1} \sin \delta_{t}+\Omega_{3}-\Omega_{2} \sin \delta_{t}-\Omega_{4}\right) \\
J_{r} q\left(\Omega_{1}-\Omega_{2}\right) \cos \delta_{t}
\end{array}\right]
$$

Gyroscopic torque vector manifests itself in a form of a rotation around unwanted axis, which is perpendicular to the axis of the propulsor and the axis around which the wanted rotation is achieved [34]. Therefore, the model should also include the gyroscopic effect resulting from the rotation of nacelles $\left(M_{1}, M_{2}\right)$ by the $\delta_{t}$ angle. However, during the transition phase, the angular speed of the rotors $\Omega_{1}=\Omega_{2}$ and rotation angles of nacelles are also the same, whereas the direction of propellers rotation is opposite. Thus, the gyroscopic moments originating from the rotors $M_{1}, M_{2}$ during the rotation by the $\delta_{t}$ angle are canceled.

The aerodynamic moment $\bar{M}_{A}$ in (22) is composed of the moment defined by the dimensionless aerodynamic coefficients and the moment resulting due to the difference in position of the centre of gravity and the centre of pressure:

$$
\begin{aligned}
\bar{M}_{A}= & {\left[\begin{array}{l}
l_{A} \\
m_{A} \\
n_{A}
\end{array}\right] } \\
= & {\left[\begin{array}{c}
\bar{q} S b C_{l} \\
\bar{q} S c C_{m} \\
\bar{q} S b C_{n}
\end{array}\right] } \\
& +\left[\begin{array}{c}
\left(c g_{Z}-\operatorname{aerp} p_{Z}\right) F_{X_{A}}-\left(c g_{X}-\operatorname{aerp} p_{X}\right) F_{Z_{A}} \\
\left(c g_{X}-\operatorname{aerp} p_{X}\right) F_{Y_{A}}
\end{array}\right]
\end{aligned}
$$

where $\bar{q}=0.5 \rho V_{\alpha}^{2}$ is free-stream dynamic pressure; $\rho$ is air density; $V_{\alpha}$ is aircraft velocity with respect to air; $S$ is wing area; $b$ is wing span; $c$ is mean aerodynamic chord of wing; $C_{l}, C_{m}$, and $C_{n}$ are dimensionless moment coefficients; $c g_{j}$ is centre of gravity in $j$-axis; aerp $p_{j}$ is aeroreference point in $j$-axis $(j=x, z)$.
The specificity of the VTOL aircraft flight causes the whole flight envelope of the UAV platform to be divided into three different flight modes: VTOL, transition, and aircraft. It was achieved by means of the collective angular displacement of the two rear rotors (Figure 4). The form of dimensionless force and moment coefficients depend also on the flight phase, and therefore in the next part of the article the analysis of the model will be carried out in the individual modes.

4.2. VTOL Mode. In the case of vertical flight, there are the following conditions: the inclination angle of the rotor nacelles $\delta_{t}=90^{\circ}$, the angle of attack $\alpha=0^{\circ}$, and the sideslip angle $\beta=0^{\circ}$ (see Figure 6). The control vector, defined in (2), is reduced to the following form:

$$
\bar{U}=\left[u_{1}, u_{2}, u_{3}, u_{4}\right]^{T}
$$

From the point of view of flight mechanics, one cannot talk about the drag, side force, and lift, as in the classic airframe approach. In the VTOL mode two main aerodynamic effects are taken into consideration. One concerns how thrust is generated while the other deals with the drag force, which is always opposite to the movement direction in $z$-axis.

The aerial drag force is expressed in the same way as in (21) with the aircraft velocity reduced to the vertical speed $W$.

$$
\begin{aligned}
\bar{F}_{B} & =\left[\begin{array}{c}
F_{X} \\
F_{Y} \\
F_{Z}
\end{array}\right]=\left[\begin{array}{c}
0 \\
0 \\
4 \\
\sum_{i=1} f_{i}
\end{array}\right]+\left[\begin{array}{c}
0 \\
0 \\
-D
\end{array}\right] \\
& =\left[\begin{array}{c}
0 \\
0 \\
\sum_{i=1}^{4} f_{i}
\end{array}\right]+\left[\begin{array}{c}
0 \\
0 \\
-\frac{1}{2} \rho W^{2} S C_{D}
\end{array}\right]
\end{aligned}
$$


In vertical flight the VTOL airframe structure produces a large aerial drag; therefore this force component has significantly large values, compared to the classic multirotor configuration.

The moments acting on the aircraft during the flight in the VTOL mode describe (22), (24), and (25); however aerodynamic moments in (25) are equal to zero $\left(l_{A}=m_{A}=\right.$ $\left.n_{A}=0\right)$.

4.3. Transition Mode. During the transition phase (see Figure 6), the main role in the generation of translational motion is played by the interaction between the angular speed of rotors $\Omega_{i}(i=1,2,3,4)$ and deflection of the rotor nacelles $\delta_{t}$, while the impact of the control surfaces is secondary. Only at speeds close to stall velocity their participation becomes significant. Therefore the control vector takes the following form:

$$
\bar{U}=\left[u_{1}, u_{2}, u_{3}, u_{4}, \delta_{t}\right]^{T}
$$

The forces acting on the VTOL aircraft are determined by (19)-(21). However, the dimensionless force coefficients $C_{D}$, $C_{Y}$, and $C_{L}$ have the following form:

$$
\begin{aligned}
& C_{D}=C_{D 0}(\alpha, \beta)_{q=\delta_{e}=0}+C_{D q}(\alpha) \frac{q c}{2 V}+C_{D \delta_{t}}(\alpha) \delta_{t} \\
& C_{Y}=C_{Y 0}(\beta)_{p=r=\delta_{r}=\delta_{a}=0}+C_{Y p}(\alpha) \frac{p b}{2 V}+C_{Y r}(\alpha) \frac{r b}{2 V} \\
& C_{L}=C_{L 0}(\alpha, \beta)_{q=\delta_{e}=0}+C_{L q}(\alpha) \frac{q c}{2 V}+C_{L \delta_{t}}(\alpha) \delta_{t}
\end{aligned}
$$

The moments are described by (22)-(25), but the dimensionless moment coefficients $C_{l}, C_{m}$, and $C_{n}$ take the form of

$$
\begin{aligned}
C_{l}= & C_{l 0}(\alpha, \beta)_{p=r=\delta_{r}=\delta_{a}=0}+C_{l p}(\alpha) \frac{p b}{2 V}+C_{l r}(\alpha) \frac{r b}{2 V} \\
C_{m}= & C_{m 0}(\alpha)_{q=\delta_{e}=0}+C_{m q}(\alpha) \frac{q c}{2 V}+C_{m \delta_{t}}(\alpha) \delta_{t} \\
C_{n}= & C_{n 0}(\alpha, \beta)_{p=r=\delta_{r}=\delta_{a}=0}+C_{n p}(\alpha) \frac{p b}{2 V} \\
& +C_{n r}(\alpha) \frac{r b}{2 V}
\end{aligned}
$$

Particular aerodynamic derivatives $C_{i}=C_{i}(\alpha, \beta)$ in (29) and (30) are calculated in ANSYS software using $3 \mathrm{D}$ analysis of the whole airframe.

4.4. Aircraft Mode. At this flight regime the aircraft has gained enough velocity (above the stall speed) to generate aerodynamic forces to lift and control the flight. In this mode, the VTOL aircraft behaves like a common airplane and the control vector contains the following variables:

$$
\bar{U}=\left[u_{t h t l}, \delta_{e}, \delta_{r}, \delta_{a}\right]^{T}
$$

where $u_{t h t l}=u_{1}+u_{2}$.

When the platform reaches the minimum cruising speed, the motors $M_{3}$ and $M_{4}$ are switched off, the inclination angle of the rotor nacelles is set to $0\left(\delta_{t}=0^{\circ}\right)$, and the driving force is given only by motors $M_{1}$ and $M_{2}$ (see Figure 6).

The forces acting in the aircraft mode are determined by (19)-(21); however the dimensionless force coefficients $C_{D}$, $C_{Y}$, and $C_{L}$ have the following form:

$$
\begin{aligned}
C_{D}= & C_{D 0}(\alpha, \beta)_{q=\delta_{e}=0}+C_{D q}(\alpha) \frac{q c}{2 V}+C_{D \delta_{e}}(\alpha) \delta_{e} \\
C_{Y}= & C_{Y 0}(\beta)_{p=r=\delta_{r}=\delta_{a}=0}+C_{Y p}(\alpha) \frac{p b}{2 V}+C_{Y r}(\alpha) \frac{r b}{2 V} \\
& +C_{Y \delta_{r}}(\alpha) \delta_{r}+C_{Y \delta_{a}}(\alpha) \delta_{a} \\
C_{L}= & C_{L 0}(\alpha, \beta)_{q=\delta_{e}=0}+C_{L q}(\alpha) \frac{q c}{2 V}+C_{L \delta_{e}}(\alpha) \delta_{e}
\end{aligned}
$$

The moments are described by (22)-(25), but the dimensionless moment coefficients $C_{l}, C_{m}$, and $C_{n}$ take the form of

$$
\begin{aligned}
C_{l}= & C_{l 0}(\alpha, \beta)_{p=r=\delta_{r}=\delta_{a}=0}+C_{l p}(\alpha) \frac{p b}{2 V}+C_{l r}(\alpha) \frac{r b}{2 V} \\
& +C_{l \delta_{r}}(\alpha, \beta) \delta_{r}+C_{l \delta_{a}}(\alpha, \beta) \delta_{a} \\
C_{m}= & C_{m 0}(\alpha)_{q=\delta_{e}=0}+C_{m q}(\alpha) \frac{q c}{2 V}+C_{m \delta_{e}}(\alpha) \delta_{e} \\
C_{n}= & C_{n 0}(\alpha, \beta)_{p=r=\delta_{r}=\delta_{a}=0}+C_{n p}(\alpha) \frac{p b}{2 V} \\
& +C_{n r}(\alpha) \frac{r b}{2 V}+C_{n \delta_{r}}(\alpha, \beta) \delta_{r} \\
& +C_{n \delta_{a}}(\alpha, \beta) \delta_{a}
\end{aligned}
$$

As in the two previous flight modes, the individual dimensionless forces and moments coefficients $C_{i}=C_{i}(\alpha, \beta)$ in (32), (33) are calculated in the ANSYS software using $3 \mathrm{D}$ CFD analysis of the entire airframe.

\section{CFD Simulations}

In order to evaluate the aerodynamic performance of designed UAV, the series of computational fluid dynamics simulations (CFD) were performed in the individual flight modes. For that purpose ANSYS Workbench 17.2 environment was used. The geometry file was imported into SpaceClaim software in which it was prepared for subsequent processing (geometry repair and optimization for CFD analysis). Since nonsymmetrical interactions were to be analysed like side wind or impact of steering surfaces position, it was necessary to create a full model of the platform without any symmetry planes. The computational domain was created on the basis of a paraboloid. The model of the UAV was placed in the centre of the domain $(0,0,0)$ in order to seamlessly determine all desired coefficients. The domain itself extended from $-20 \mathrm{~m}$ to $30 \mathrm{~m}$ in X (platform longitudinal) direction and from $-30 \mathrm{~m}$ to $30 \mathrm{~m}$ in $\mathrm{Y}$ and $\mathrm{Z}$ directions. Such prepared geometry was transferred into ANSYS Meshing software. Due to the complexity of the geometry an unstructural mesh was generated consisting of tetrahedral elements. In order to correctly simulate gradients 
at the surfaces (the $y+<1$ condition) the inflation (boundary layer) consisting of prism elements was generated. Next, the grid-independence study was performed. As the optimal solution $16 \mathrm{mln}$ elements mesh was chosen with inflation layer consisting of 15 sublayers. Such prepared computational domain was exported into Fluent solver.

Since low flight velocities (low Reynolds number) were to be simulated and therefore no sudden changes in pressure were expected, the pressure-based solver was chosen [35]. In order to determine all force and moment coefficients as well as the dynamic interactions the simulations were made in the steady state and transient. However the data presented in this section concern only the steady state cases so as the interested reader could compare it more easily with other publications.

All physicochemical properties of air were constant and averaged for the weather conditions at which the UAV will be tested. Nowadays one may find publications in which CFD analysis of the UAVs is performed using one-equation turbulence model (Spallart-Allmars) or two-equation turbulence model (usually SST k- $\omega$ ) $[36,37]$. However, in order to achieve high accuracy of obtained results the four-equation Transitional SST turbulence model [38, 39] was employed in the presented research. The major disadvantage of this decision was significantly longer times of calculations. The Langtry-Menter four-equation Transitional SST turbulence model $[40,41]$ originates from the SST k- $\omega$ turbulence model $[42,43]$. It couples its transport equations with the intermittency equation and the equation for the transition onset criteria in terms of momentum-thickness Reynolds number. The transport equation for the intermittency $\gamma$ is defined as follows [35]:

$$
\begin{aligned}
\frac{\partial(\rho \gamma)}{\partial t}+\frac{\partial\left(\rho U_{j} \gamma\right)}{\partial x_{j}}= & P_{\gamma 1}-E_{\gamma 1}+P_{\gamma 2}-E_{\gamma 1} \\
& +\frac{\partial}{\partial x_{j}}\left[\left(\mu+\frac{\mu_{t}}{\sigma_{\gamma}}\right) \frac{\partial \gamma}{\partial x_{j}}\right]
\end{aligned}
$$

and for the transition momentum-thickness Reynolds number:

$$
\begin{aligned}
& \frac{\partial\left(\rho \mathrm{R}_{\theta \mathrm{t}}\right)}{\partial \mathrm{t}}+\frac{\partial\left(\rho \mathrm{U}_{\mathrm{j}} \mathrm{R} \widetilde{\mathrm{e}}_{\theta \mathrm{t}}\right)}{\partial \mathrm{x}_{\mathrm{j}}} \\
& \quad=P_{\theta t}+\frac{\partial}{\partial x_{j}}\left[\sigma_{\theta t}\left(\mu+\mu_{t}\right) \frac{\mathrm{R}_{\theta t}}{\partial x_{j}}\right]
\end{aligned}
$$

The transition model interacts with the SST turbulence model by modification of the k-equation [35]:

$$
\begin{aligned}
\frac{\partial}{\partial t} & (\rho k)+\frac{\partial}{\partial x_{i}}\left(\rho k u_{i}\right) \\
& =G_{k}{ }^{*}-Y_{k}{ }^{*}+S_{k}{ }^{*}+\frac{\partial}{\partial x_{j}}\left(\Gamma_{k} \frac{\partial k}{\partial x_{j}}\right)
\end{aligned}
$$

Finally, the $\omega$-equation has the following form [35]:

$$
\begin{aligned}
& \frac{\partial}{\partial t}(\rho \omega)+\frac{\partial}{\partial x_{j}}\left(\rho \omega u_{j}\right) \\
& \quad=G_{\omega}-Y_{\omega}+D_{\omega}+S_{\omega}+\frac{\partial}{\partial x_{j}}\left(\Gamma_{\omega} \frac{\partial \omega}{\partial x_{j}}\right)
\end{aligned}
$$

Due to the limited computational power Semi-Implicit Method for Pressure Linked Equations (SIMPLE) was used instead of coupled pressure-velocity coupling algorithm. All discretization schemes were set to the second-order upwind whereas the gradients were evaluated using GreenGauss Node-Based method. As the convergence criteria the values of all scaled residuals smaller than $1 \mathrm{e}-03$ were assumed. Simultaneously the value of lift and drag coefficients were monitored. The iterations were continued if these values did not achieve the constant level. Three different boundary conditions were used in simulations. The peripheral of paraboloid was set as velocity inlet for which direction vector and velocity magnitude were set. The back surface of paraboloid was set as pressure outlet. Finally, the surface of the UAV was treated as wall with no slip condition.

In order to fulfil all requirements for which the UAV was designed it should be characterized by high aerodynamic efficiency. This feature will allow it not only to take off and land at any desired location but also, more importantly, to travel for long distances. As one should expect the construction modification of a classical plane for hovering mode of flight will always influence unfavourably the aerodynamic properties of the platform. Figure 9 presents the lift and drag coefficients as the functions of angle of attack (AoA) for $\operatorname{Re}=237$ 000. Although the maximal lift coefficient is low comparing to other conceptual studies [44], especially dedicated for the same purpose [16], the designed UAV has satisfactory parameters from the project aims point of view. The zero lift condition appears for AoA equal to $-1^{\circ}$ whereas the stall point is achieved for $12^{\circ}$. The generated lift and drag are sufficient to travel for the intended distance.

Figure 10 presents the pitching moment. It changes its value from positive to negative at approximately the same angle of attack as for the zero lift condition. After this point it decreases steadily up to the stall region. The negative slope indicates positive static longitudinal stability of the UAV.

Figure 11 represents lift to drag ratio as the function of angle of attack. Moreover the impact of side wind is analysed. As one may notice the designed platform behaves stably despite unfavourable conditions. Although the impact of side wind on both the lift and drag is almost unnoticeable when considered separately, if these two parameters are combined some decrease in efficiency is visible. As it should be expected the biggest differences were noticed for $10^{\circ}$ side wind (maximal investigated value) for which lift to drag ratio decreased by $27 \%, 22 \%$, and $18 \%$ for $0^{\circ}, 2^{\circ}$, and $4^{\circ}$, respectively. What is interesting in the case of $5^{\circ}$ side wind and AoA beyond $8^{\circ}$ is that the lift to drag ratio increased by $1 \%$. 


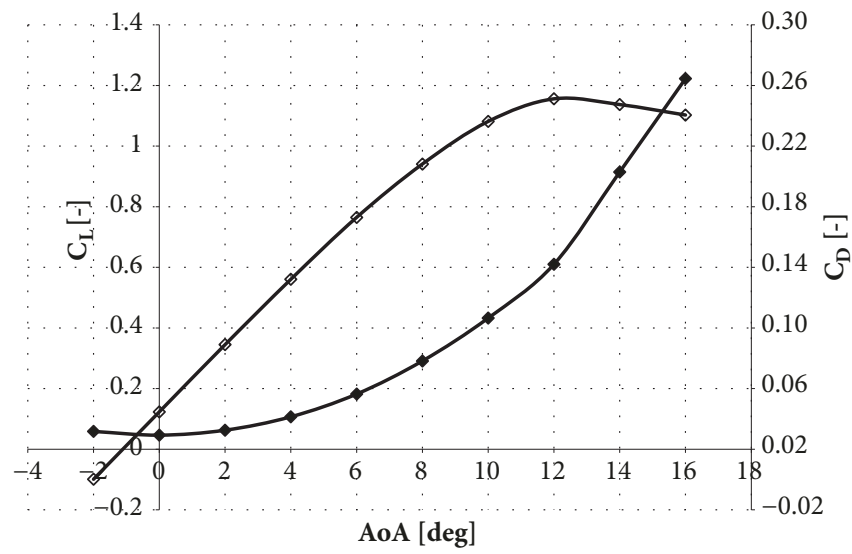

$\neg$ Lift coefficient

FIGURE 9: Lift and drag coefficients versus angle of attack (AoA) for the final UAV design $(\operatorname{Re}=237000)$.

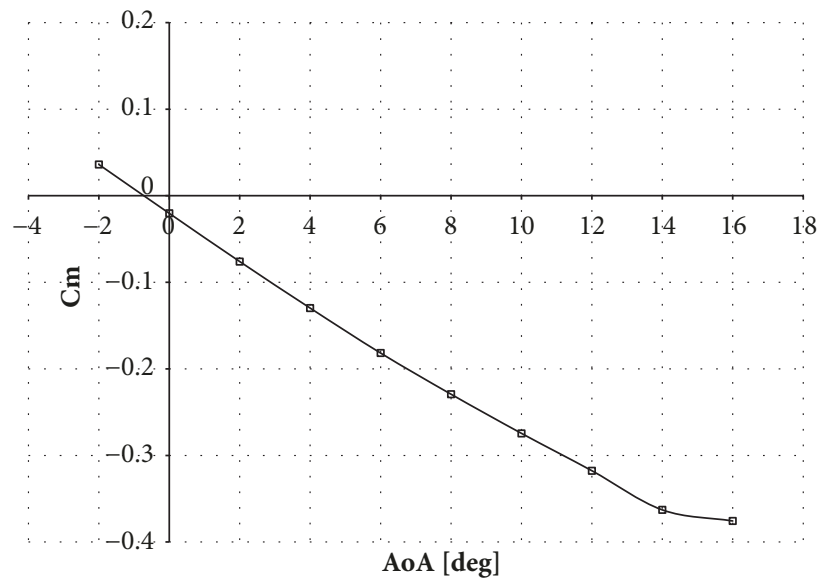

FIGURE 10: Pitching moment coefficient versus AoA for the final UAV design $(\operatorname{Re}=237000)$.

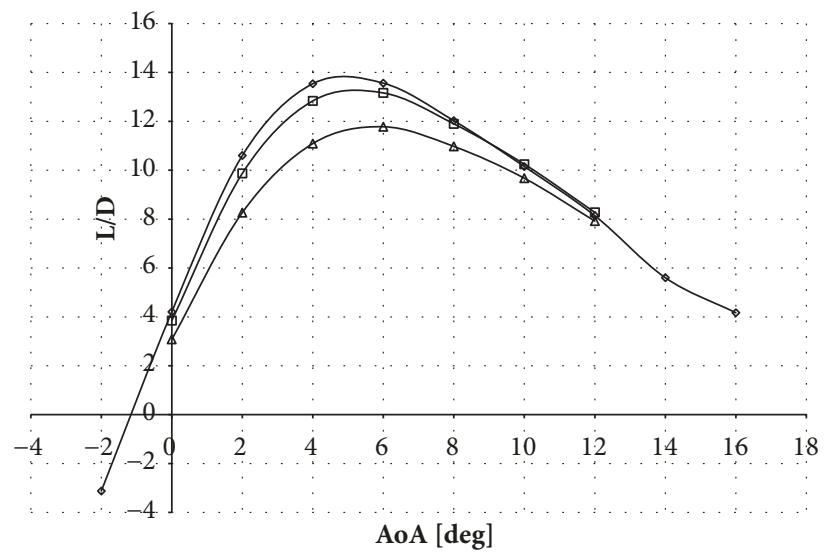

$$
\begin{aligned}
& \text { - Side wind }(0 \mathrm{deg}) \\
& \text { - Side wind }(5 \mathrm{deg}) \\
& \text { - Side wind (10 deg) }
\end{aligned}
$$

FIGURE 11: Impact of the side wind onto lift to drag ratio for the final UAV design $(\operatorname{Re}=237000)$. 


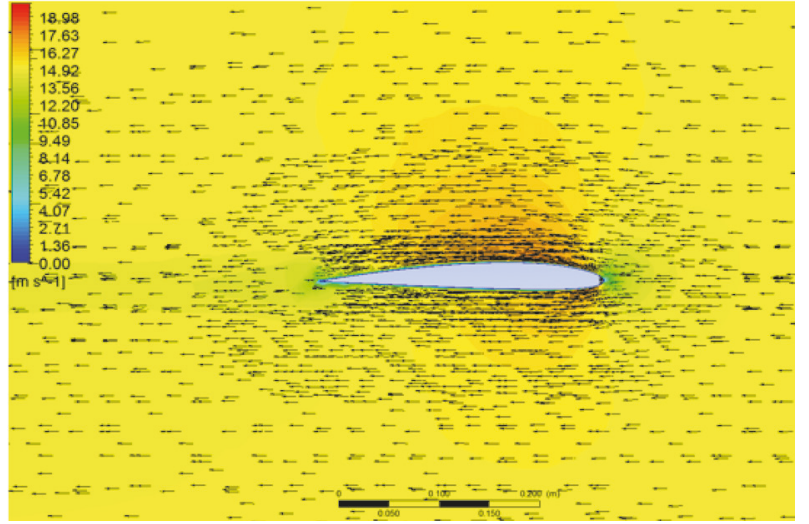

(a)

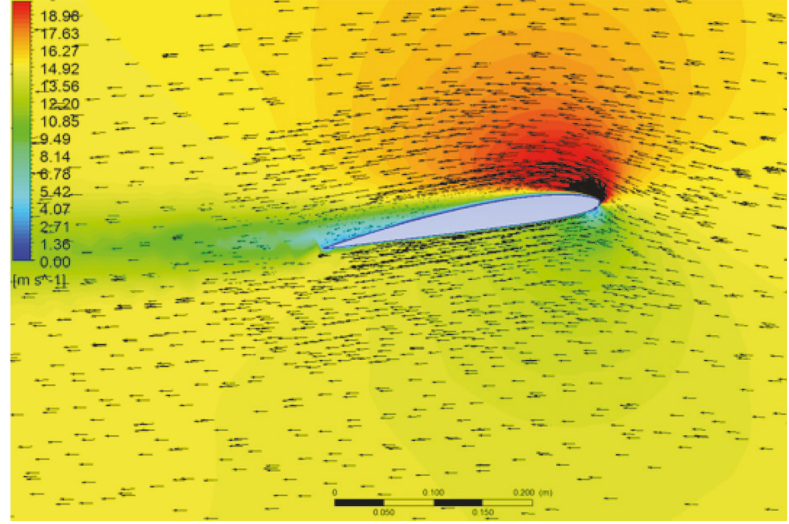

(b)

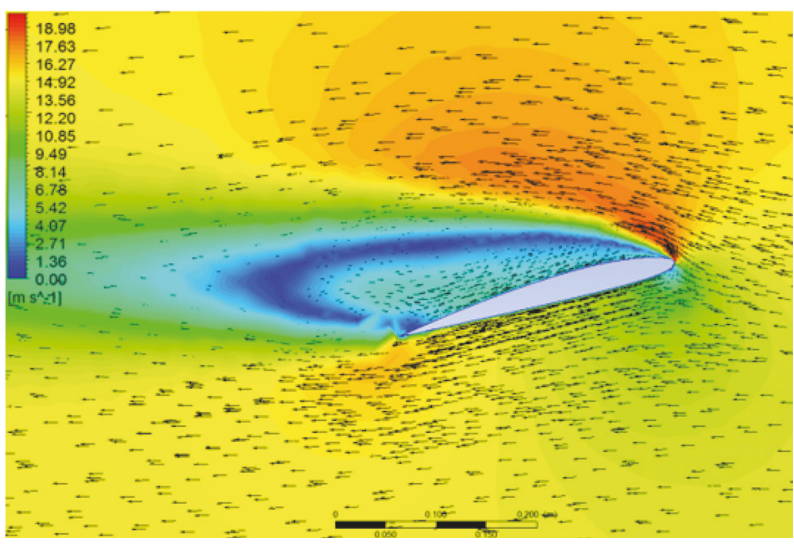

(c)

FIGURE 12: Velocity contour plots and wind velocity vectors $(\mathrm{Re}=237000)$ for $(\mathrm{a}) 0^{\circ} \mathrm{AoA}$, (b) $12^{\circ} \mathrm{AoA}$, and $(\mathrm{c}) 16^{\circ} \mathrm{AoA}$.

Figure 12 presents the process of wind streamlined detachment from the wing surface during the increase of AoA. For the $0^{\circ}$ AoA (Figure $12(\mathrm{a})$ ) air steadily flows around the wing. A region of higher velocity is visible above the wing generating small lift force (Figure 9). Moreover, a thin laminar layer may be noticed around solid surface. When the AoA is increased up to $12^{\circ}$ (Figure 12(b)) which corresponds to the maximal lift force (Figure 9) one may notice a region of significantly higher velocity above the wing. Simultaneously first streamlines of air start to detach at the end of the surface. At this angle wing becomes a serious obstacle for the air flow. Finally, for AoA equal to $16^{\circ}$ the detachment is clearly visible. The lift force decreases and the drag force increases substantially.

The impact of AoA value on the vorticity is presented in Figure 13. The UAV is surrounded by an isosurface generated for $100 \mathrm{~s}^{-1}$ vorticity in order to depict the negative impact of nacelles on the lifting capabilities of the platform. The color of the isosurface represents local air velocity. At $0^{\circ}$ AoA the vortices are generated mainly at the tips of winglets and behind the nacelles and tail. When the AoA was increased up to $12^{\circ}$ some crucial changes may be noticed. The construction of nacelles in front of the wing generates vortices influencing aerodynamic performance of the wings. Significantly higher vorticity is visible behind the wing, especially in the region of nacelles and winglets. Finally, at AoA equal to $16^{\circ}$ extremely high vorticity is visible especially in the case of nacelles.

The last step of the simulations involved the analysis of aerodynamic properties of the VTOL during hovering and transition stage of flight. In these simulation the impact of propeller action on the air velocity profile around the wings also had to be included. It was done by generation of four volumes of fluid which corresponded to the volumes created during rotational movement of propellers. Next, for each volume, appropriate momentum source was defined within the solver. As in the previous cases, all simulations were made in the steady state. The simulations were performed for various ranges of velocities, tilt angles, and thrust values in order to determine force and moments coefficients introduced in the fourth chapter of this work. An exemplary result is presented in Figure 14, in which the vortices generated by the propellers are depicted. Of course the disturbance of air flow below the wing resulting from the action of front propellers has a negative influence on the overall aerodynamic properties of the platform. Therefore, the transition phase demands the optimization of the flight scenario which will include the thrust of propulsion system and angle of attack value as well as flight velocity. 


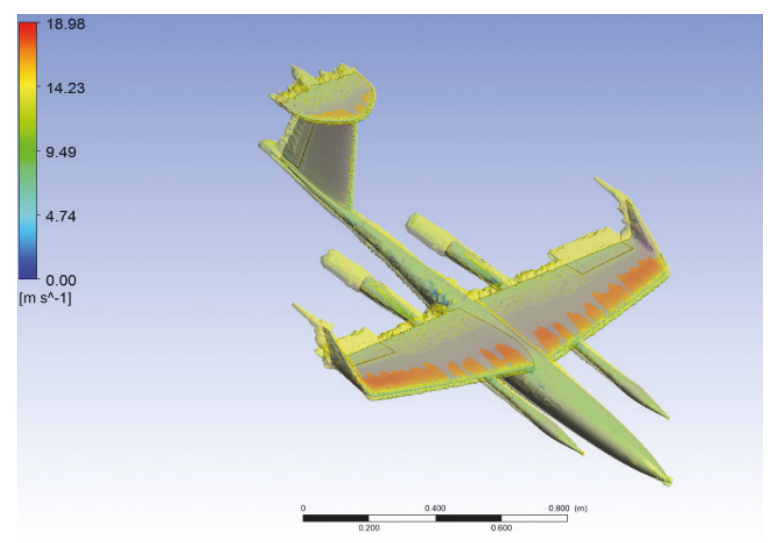

(a)

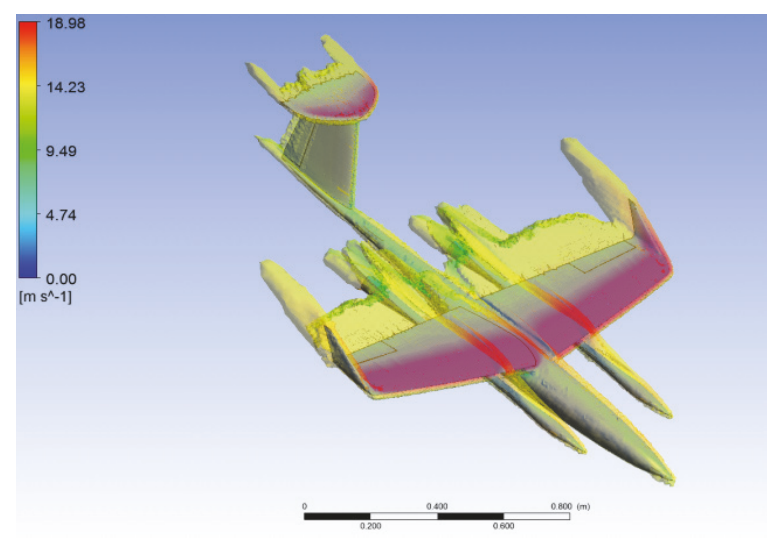

(b)

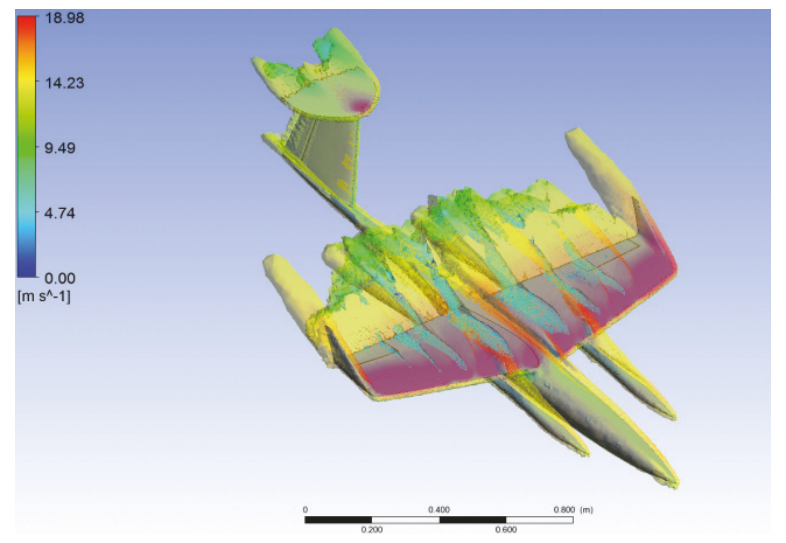

(c)

FiguRE 13: Isosurface of vorticity $(\mathrm{Re}=237000)$ for $100 \mathrm{~s}^{-1}$ (the color of isosurface represents wind velocity) for: (a) $0^{\circ} \mathrm{AoA}$, (b) $12^{\circ} \mathrm{AoA}$, and (c) $16^{\circ}$ AoA.

\section{Conclusions}

In this paper the design process of a VTOL aircraft was presented. The evolution of the design from three support points platform with ducted fuselage propeller into four support points " $H$ " configuration was discussed. An unusual configuration of propellers was proposed, namely, all four propellers directed downward during hovering mode and propellers directed backward (pushing configuration) in the standard flight mode. As it was proven, such design is more efficient in aerodynamics terms. The platform has fixedwing configuration. The rotors are mounted on beams placed below wings. The front rotors have fixed position; however their construction allows them to fold due to action of drag. In the airplane mode they are switched off, because they only disturb the flow of air streams, without significantly increasing the lift force generated on the wing. The rear rotors are mounted on nacelles that ensure the thrust vectoring of engines. This provides their usage in both vertical and horizontal flight. The advantage of proposed approach is an ability to rotate only the rotors themselves, not the whole wing. The proposed solution makes the aircraft more robust to cross winds. The mathematical model of each of flight modes was proposed and discussed. Finally the results of
CFD simulations were presented for the final design.The degree of complexity of the aerodynamic properties of such UAV obviously makes it impossible to control it in the open loop system and enforces regulation in a closed loop system with feedback. Due to nonlinearities and instability resulting from the stall condition, the transition mode is particularly dangerous and demanding, in terms of reliability of the control system. CFD analysis results presented in this paper have given a broad view on the aerodynamic properties of the aircraft, in particular on the stall effect.

Looking prospectively, such constructions could be used in an urban environment, where the use of a runway is not possible. Such platforms will be useful when high maneuverability in a limited area is demanded and the flight time should be relatively long (above one hour), which is unachievable in the case of conventional multirotor carriers.

\section{Data Availability}

All data used to support the findings of this study are included within the article. The CFD data used to support the findings of this study are available from the corresponding author upon request. 


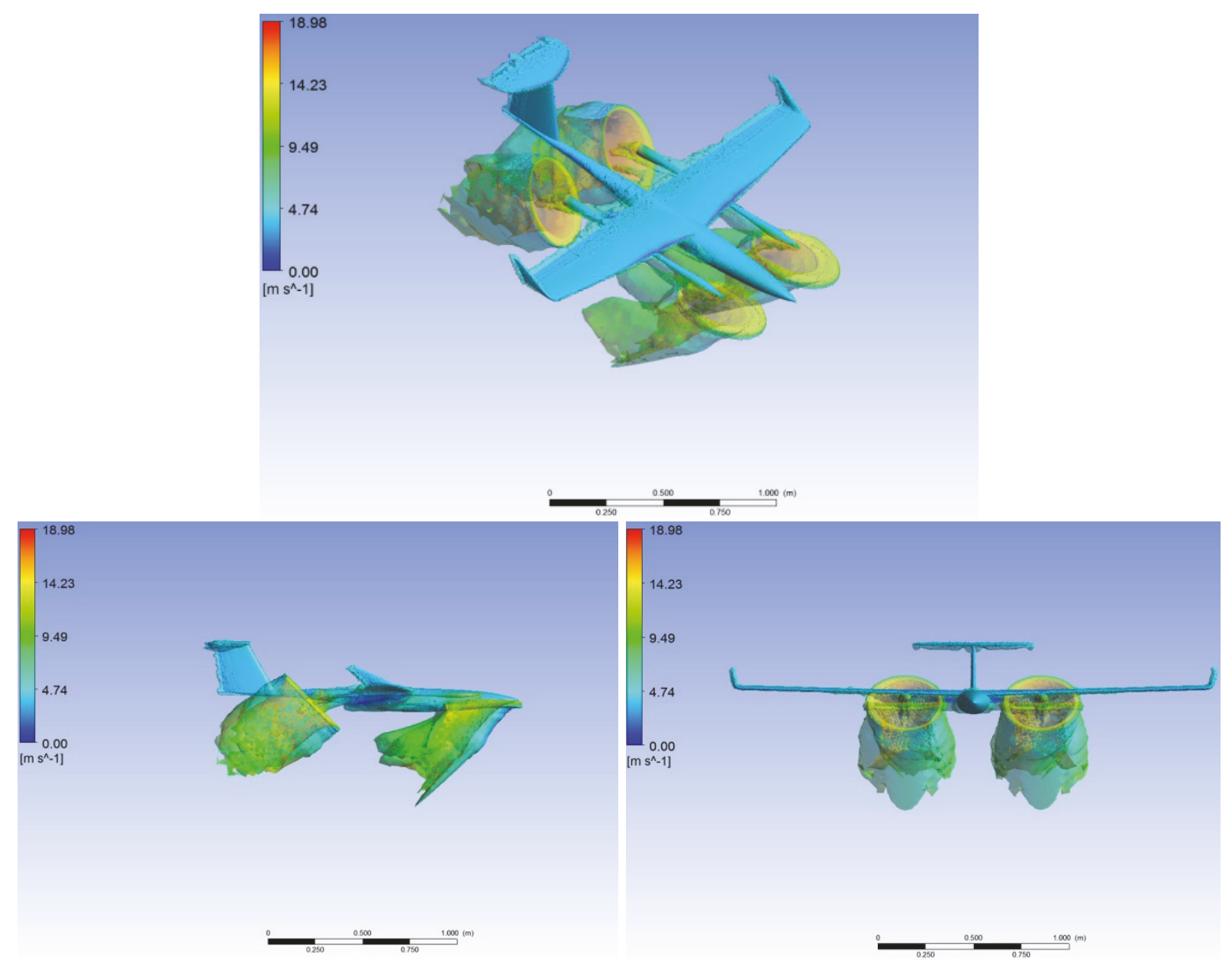

FIGURE 14: Isosurface of vorticity $(\mathrm{Re}=77000)$ for $100 \mathrm{~s}^{-1}$ (the color of isosurface represents wind velocity) for AoA $=0^{\circ}$; tilt of the rear rotor nacelles $\delta_{t}=45^{\circ}$.

\section{Conflicts of Interest}

The authors declare that there are no conflicts of interest regarding the publication of this paper.

\section{Acknowledgments}

This work has been granted by the Polish Ministry of Science and Higher Education from funds of European Union and national funds for years 2017-2018 [grant No. MNiSW/2017/110/DIR/NN2]. The computational fluid simulations were performed in ANSYS environment using IT infrastructure of GeCONiI [grant NCBiR POIG 02.03.01-24099].

\section{References}

[1] R. Austin, Unmanned Aircraft Systems -UAVS Design, Development and Deployment, John Wiley \& Sons, 2010.

[2] M. Logan, T. Vranas, M. Motter, Q. Shams, and D. Pollock, Technology Challenges in Small UAV Development, Infotech@Aerospace, 2005.

[3] A. C. Watts, V. G. Ambrosia, and E. A. Hinkley, "Unmanned aircraft systems in remote sensing and scientific research:
Classification and considerations of use," Remote Sensing, vol. 4, no. 6, pp. 1671-1692, 2012.

[4] K. P. Valavanis, Advances in Unmanned Aerial Vehicles, vol. 33, Springer Netherlands, Dordrecht, 2007.

[5] P. Castillo, R. Lozano, and A. E. Dzul, Modelling and Control of Mini-flying Machines, Springer-Verlag, 2005.

[6] K. Nonami, F. Kendoul, S. Suzuki, W. Wang, and D. Nakazawa, "Autonomous flying robots: Unmanned aerial vehicles and micro aerial vehicles," Autonomous Flying Robots: Unmanned Aerial Vehicles and Micro Aerial Vehicles, pp. 1-329, 2010.

[7] R. Czyba, G. Szafrański, W. Janusz, M. Niezabitowski, A. Czornik, and M. Błachuta, "Concept and realization of unmanned aerial system with different modes of operation," in Proceedings of the 10Th International Conference on Mathematical Problems in Engineering, Aerospace And Sciences: Icnpaa 2014, pp. 261-270, Narvik, Norway, 2014.

[8] K. Dalamagkidis, "Aviation history and unmanned flight," Handbook of Unmanned Aerial Vehicles, pp. 57-81, 2015.

[9] https://www.sesarju.eu/U-Space.

[10] https://www.skyguide.ch/en/events-media-board/u-space-livedemonstration/.

[11] R. Czyba, G. Szafranski, A. Rys, G. Szafrański, and A. Ryś, "Design and control of a single tilt tri-rotor aerial vehicle," Journal of Intelligent Robotic Systems, vol. 84, pp. 53-66, 2016. 
[12] R. Czyba, G. Szafranski, M. Janik, K. Pampuch, and M. Hecel, "Development of co-axial Y6-Rotor UAV - design, mathematical modeling, rapid prototyping and experimental validation," in Proceedings of the 2015 International Conference on Unmanned Aircraft Systems (ICUAS), pp. 1102-1111, Denver, CO, USA, June 2015.

[13] F. Çakıcı and M. K. Leblebicioğlu, "Design and analysis of a mode-switching micro unmanned aerial vehicle," International Journal of Micro Air Vehicles, vol. 8, no. 4, pp. 221-229, 2016.

[14] G. R. Flores-Colunga and R. Lozano-Leal, "A nonlinear control law for hover to level flight for the Quad Tilt-rotor UAV," IFAC Proceedings Volumes, vol. 47, no. 3, pp. 11055-11059, 2014.

[15] Y. O. Aktas, U. Ozdemir, and Y. Dereli, "Rapid Prototyping of a Fixed-Wing VTOL UAV for Design Testing," JournalofIntelligentRoboticSystems, vol. 84, pp. 639-664, 2016.

[16] D. Orbea, J. Moposita, W. G. Aguilar, M. Paredes, R. P. Reyes, and L. Montoya, "Vertical take off and landing with fixed rotor," in Proceedings of the 2017 CHILEAN Conference on Electrical, Electronics Engineering, Information and Communication Technologies (CHILECON), pp. 1-6, Pucon, October 2017.

[17] L. Zhong, H. Yuqing, Y. Liying et al., "Control techniques of tilt rotor unmanned aerial vehicle systems: A review," Chinese Journal of Aeronautics, vol. 30, no. 1, pp. 135-148, 2017.

[18] A. M. Stoll, E. V. Stilson, J. Bevirt, and P. Sinha, "A Multifunctional Rotor Concept for Quiet and Efficient VTOL Aircraft," in Proceedings of the 2013 Aviation Technology, Integration, and Operations Conference, Los Angeles, CA, USA.

[19] J.-T. Zou and Z.-Y. Pan, "The development of tilt-rotor unmanned aerial vehicle," Transactions of the Canadian Society for Mechanical Engineering, vol. 40, no. 5, pp. 909-921, 2016.

[20] B. Defense, V-22 osprey, Space and Security.

[21] E. Cetinsoy, S. Dikyar, C. Hancer et al., "Design and construction of a novel quad tilt-wing UAV," Mechatronics, vol. 22, no. 6, pp. 723-745, 2012.

[22] R. Udroiu and M. Blaj, "Conceptual design of a vtol remotely piloted aircraft for emergency missions," Scientific Research and Education in the Air Force, vol. 18, no. 1, pp. 207-214, 2016.

[23] R. Park, Arcturus uav upgrades the jump15 vtol uav, Airlines \& Aviation, Aerospace \& Defense, December 2014.

[24] M. Hochstenbach, C. Notteboom, B. Theys, and J. De Schutter, "Design and control of an unmanned aerial vehicle for autonomous parcel delivery with transition from vertical takeoff to forward flight - VertiKUL, a quadcopter tailsitter," International Journal of Micro Air Vehicles, vol. 7, no. 4, pp. 395-405, 2015.

[25] R. Ritz and R. D’Andrea, "A global controller for flying wing tailsitter vehicles," in Proceedings of the 2017 IEEE International Conference on Robotics and Automation (ICRA), pp. 2731-2738, Singapore, Singapore, May 2017.

[26] B. Theys, G. Dimitriadis, P. Hendrick, and J. De Schutter, "Influence of propeller configuration on propulsion system efficiency of multi-rotor Unmanned Aerial Vehicles," in Proceedings of the 2016 International Conference on Unmanned Aircraft Systems (ICUAS), pp. 195-201, Arlington, VA, USA, June 2016.

[27] https://noveltyrpas.com/ogar-mk2/.

[28] Y. Jiang, B. Zhang, and T. Huang, "CFD study of an annularducted fan lift system for VTOL aircraft," Aerospace, vol. 2, no. 4, pp. 555-580, 2015.

[29] B. L. Stevens, F. L. Lewis, and E. N. Johnson, Aircraft Control and Simulation: Dynamics, Controls Design, and Autonomous Systems, Wiley-Blackwell, 3rd edition, 2015.
[30] A. Abhishek, "Tripathi, Six-rotor UAV helicopter dynamics and control: theory and simulation," International Journal of Advanced Research in Electrical Electronics and Instrumentation Engineering, vol. 2, no. 11, pp. 5747-5755, 2013.

[31] Z. Chen, Z. Peng, and F. Zhang, "Attitude control of coaxial tri-rotor UAV based on Linear Extended State Observer," in Proceedings of the 2014 26th Chinese Control And Decision Conference (CCDC), pp. 4204-4209, Changsha, China, May 2014.

[32] G. Szafranski, R. Czyba, and M. Blachuta, "Modeling and identification of electric propulsion system for multirotor unmanned aerial vehicle design," in Proceedings of the 2014 International Conference on Unmanned Aircraft Systems, ICUAS 2014, pp. 470-476, USA, May 2014.

[33] Y. Naidoo, R. Stopforth, and G. Bright, "Rotor Aerodynamic Analysis of a Quadrotor for Thrust," CSIR Pretoria South Africa, 2011.

[34] P. Shao, W. Dong, X. Sun, T. Ding, and Q. Zou, "Dynamic surface control to correct for gyroscopic effect of propellers on quadrotor," in Proceedings of the 2015 IEEE International Conference on Information and Automation (ICIA), pp. 29712976, Lijiang, China, August 2015.

[35] “Fluent User's Guide," ANSYS INC., 2016.

[36] S. G. Kontogiannis and J. A. Ekaterinaris, "Design, performance evaluation and optimization of a UAV," Aerospace Science and Technology, vol. 29, no. 1, pp. 339-350, 2013.

[37] P. D. Bravo-Mosquera, L. Botero-Bolivar, D. Acevedo-Giraldo, and H. D. Cerón-Muñoz, "Aerodynamic design analysis of a UAV for superficial research of volcanic environments," Aerospace Science and Technology, vol. 70, pp. 600-614, 2017.

[38] S. M. Aftab, A. S. Mohd Rafie, N. A. Razak, K. A. Ahmad, and X. Wang, "Turbulence Model Selection for Low Reynolds Number Flows," PLoS ONE, vol. 11, no. 4, p. e0153755, 2016.

[39] S. M. Aftab and K. A. Ahmad, "Correction: CFD study on NACA 4415 airfoil implementing spherical and sinusoidal Tubercle Leading Edge," PLoS ONE, vol. 12, no. 11, p. e0188792, 2017.

[40] R. B. Langtry and F. R. Menter, "Correlation-based transition modeling for unstructured parallelized computational fluid dynamics codes," AIAA Journal, vol. 47, no. 12, pp. 2894-2906, 2009.

[41] F. R. Menter, R. Langtry, and S. Völker, "Transition modelling for general purpose CFD codes," Flow, Turbulence and Combustion, vol. 77, no. 1-4, pp. 277-303, 2006.

[42] F. R. Menter, "Two-equation eddy-viscosity turbulence models for engineering applications," AIAA Journal, vol. 32, no. 8, pp. 1598-1605, 1994.

[43] F. R. Menter, M. Kuntz, and R. Langtry, “Ten Years of Industrial Experience with the SST Turbulence Model, Turbulence, HeatandMassTransfer," in Ten Years of Industrial Experience with the SST Turbulence Model, Turbulence, HeatandMassTransfer, K. Hanjalic, Y. Nagano, and M. Tummers, Eds., pp. 625-632, Begell House, Inc, HeatandMassTransfer, 2003.

[44] P. Panagiotou, S. Fotiadis-Karras, and K. Yakinthos, "Conceptual design of a Blended Wing Body MALE UAV," Aerospace Science and Technology, vol. 73, pp. 32-47, 2018. 


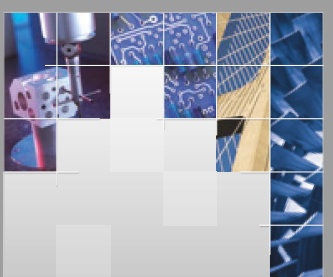

\section{Enfincering}
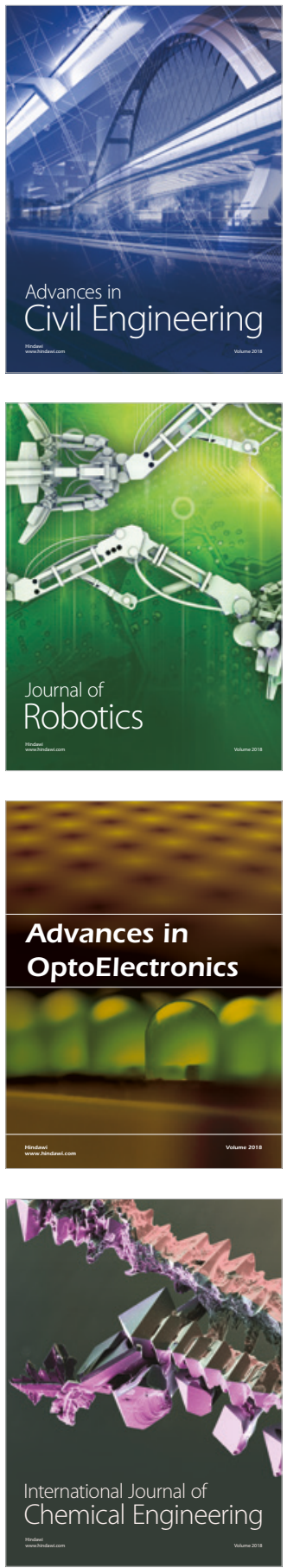

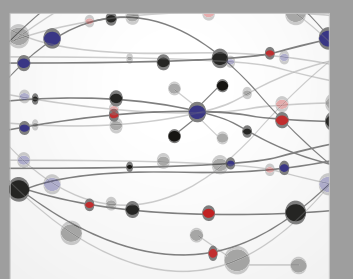

\section{Rotating \\ Machinery}

The Scientific World Journal

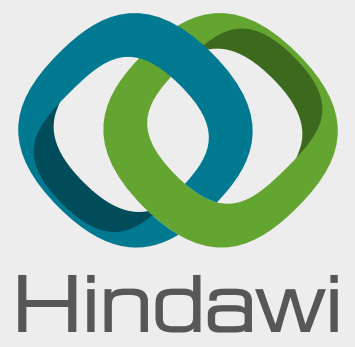

Submit your manuscripts at

www.hindawi.com
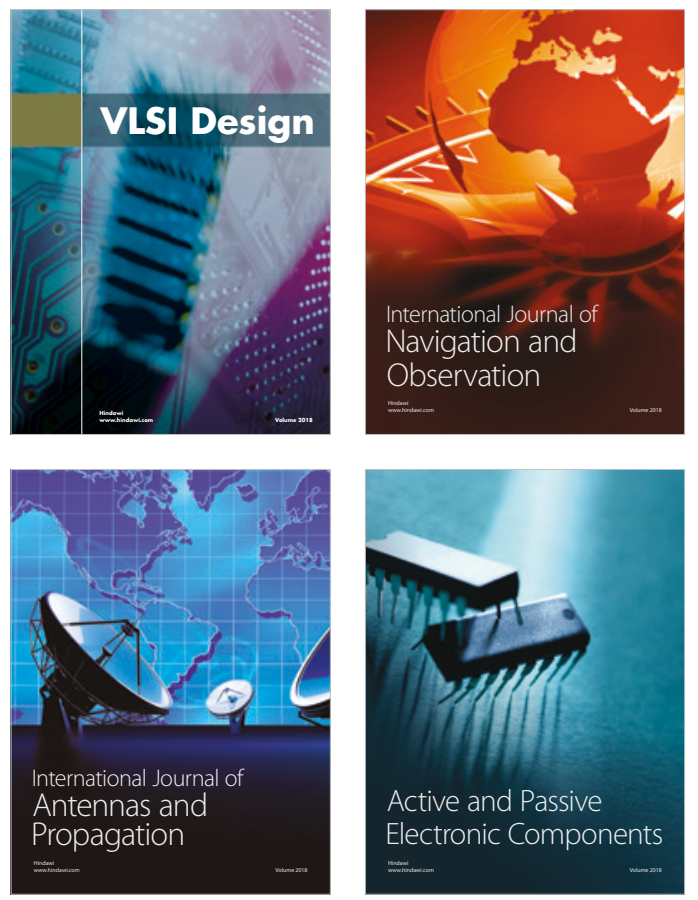
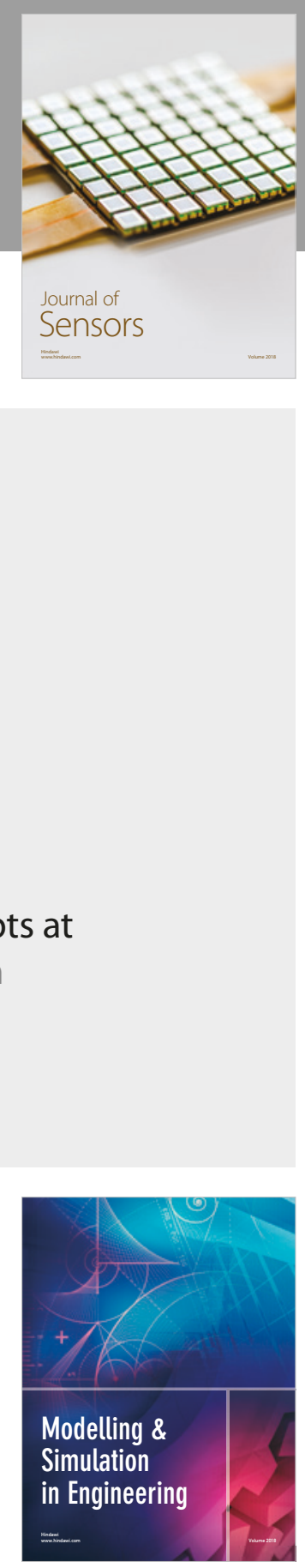

\section{Advances \\ Multimedia}
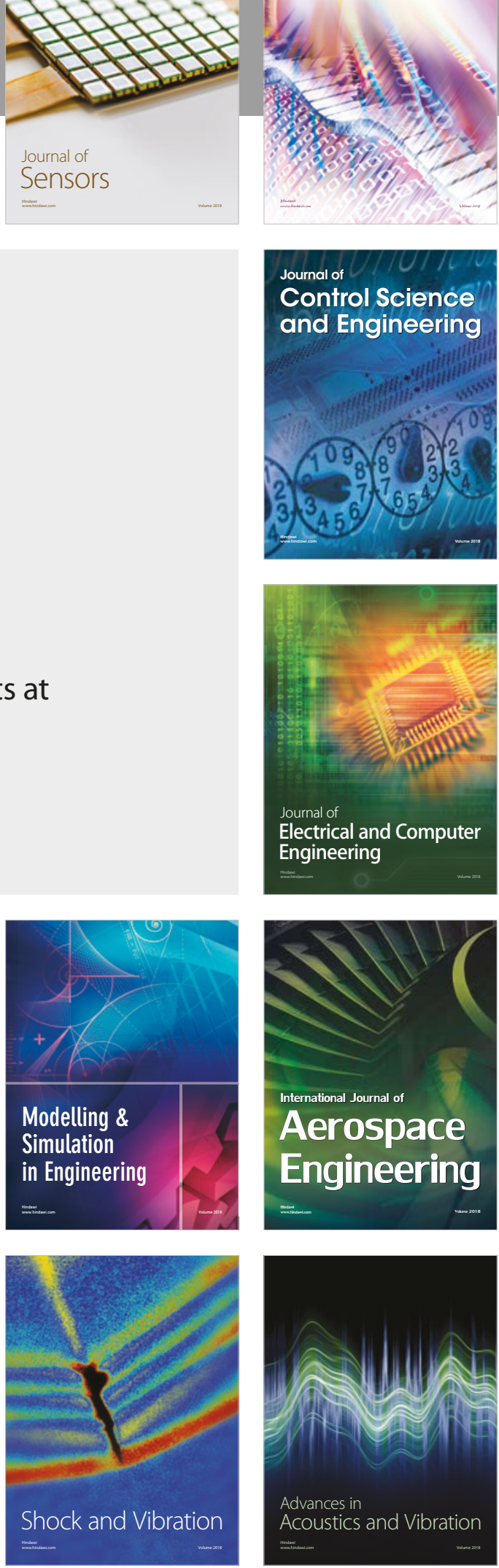